\title{
Highly Sensitive Biomolecule Detection on a Quartz Crystal Microbalance Using Gold Nanoparticles as Signal Amplification Probes
}

\author{
Nam Hyun KIM,* Taek Jin Baek,* Hyun Gyu Park,** and Gi Hun SEOnG*广 \\ * Department of Applied Chemistry, Hanyang University, Sa-1 dong 1271, Ansan 425-791, South Korea \\ **Department of Chemical \& Biomolecular Engineering, Korea Advanced Institute of Science \& Technology \\ (KAIST), Daejeon 305-701, South Korea
}

\begin{abstract}
We report here a novel strategy for the high-sensitive detection of target biomolecules with very low concentrations on a quartz crystal microbalance (QCM) device using gold nanoparticles as signal enhancement probes. By employing a streptavidin-biotin interaction as a model system, we could prepare biotin-conjugated gold nanoparticles maintaining good dispersion and long-term stability by controlling the biotin density on the surface of gold nanoparticles that have been investigated by UV-vis spectra and AFM images. These results showed that $10 \mu \mathrm{M} \mathrm{N}$-(6-[biotinamido]hexyl)-3'( 2 '-pyridyldithio)propionamide (biotin-HPDP) was the critical concentration to prevent the nonspecific aggregation of gold nanoparticles in this system. For sensing streptavidin target molecules by QCM, biotinylated BSA was absorbed on the $\mathrm{Au}$ surface of the QCM electrode and subsequent coupling of the target streptavidin to the biotin in the sensing interface followed. Amplification of the sensing process was performed by the interaction of the target streptavidin on the sensing surface with gold nanoparticles modified with $10 \mu \mathrm{M}$ biotin-HPDP. The biotinylated gold nanoparticles were used as signal amplification probes to improve the detection limit, which was $50 \mathrm{ng} / \mathrm{ml}$, of the streptavidin detection system without signal enhancement, and the calibration curve determined for the net frequency changes showed good linearity over a wide range from $1 \mathrm{ng} / \mathrm{ml}$ to $10 \mu \mathrm{g} / \mathrm{ml}$ for the quantitative streptavidin target molecule analysis. In addition, the measured dissipation changes suggested that the layer of biotin-BSA adsorbed on the Au electrode and the streptavidin layer assembled on the biotin-BSA surface were highly compact and rigid. On the other hand, the structure formed by the biotinylated gold nanoparticles on the streptavidin layer was flexible and dissipative, being elongated outward from the sensing surface.
\end{abstract}

(Received August 2, 2006; Accepted October 2, 2006; Published February 10, 2007)

\section{Introduction}

The unique optical, electronic, magnetic, and catalytic properties of metal and semiconductor nanoparticles that are coupled to biomaterials have led to a great deal of progress in bioassays, molecular diagnostics, bioelectronics, and bioengineering. ${ }^{1} \quad$ Semiconductor nanoparticles, having sizedependent tunable fluorescence and photostability, have been used as fluorescence labels for biorecognition processes. ${ }^{2}$ Biomaterial-functionalized magnetic particles have been extensively applied in biodetection systems as well as for the separation and concentration of target biomolecules. ${ }^{3}$ Among the nanoparticles, gold nanoparticles exhibit novel properties and functions for the emerging and rapidly progressing field of nanobiotechnology. By controlling the size and composition of the particles, the different plasmon absorbance bands in the visible spectral region have been widely used for the photonic detection of biorecognition processes such as DNA hybridization and antibody-antigen analysis.,5 Besides the unique optical properties of the gold nanoparticles, the particles also have been applied as the diffusional electron mediators to

$\doteqdot$ To whom correspondence should be addressed. E-mail: ghseong@hanyang.ac.kr achieve efficient electrical contacting of redox enzymes with electrodes, ${ }^{6}$ as the analytic tagged probes to give quantitative molecule-counting capabilities with a good signal-to-noise ratio, ${ }^{7,8}$ and as catalytic tags for the construction of nanoscale devices. $^{9}$

In particular, gold nanoparticles have been used as signal enhancement probes in a wide variety of fields. Mirkin and coworkers have developed a scanometric DNA array system coupled with a signal enhancement method based on gold nanoparticle-promoted reduction of silver ion. ${ }^{10}$ The sensitivity of this scanometric array detection system exceeds that of the analogous fluorophore system by two orders of magnitude. Wang et al. reported a silver-enhanced colloidal gold stripping detection strategy for detecting DNA hybridization, based on the precipitation of silver on gold nanoparticle tags and the subsequent electrochemical stripping detection of the dissolved silver. $^{11} \quad$ Keating and coworkers described ultrasensitive detection of DNA hybridization based on gold nanoparticleamplified surface plasmon resonance (SPR). ${ }^{12}$ A great improvement in the SPR response by a combination of increased surface mass, high dielectric constant of gold particles, and electromagnetic coupling between gold nanoparticles and the gold film was achieved compared to the unamplified binding event. Porter and coworkers demonstrated a surface-enhanced Raman scattering (SERS)-based 
immunoassay using gold nanoparticles modified with antibodies, ${ }^{13}$ and Fan et al. presented an oxidative gold nanoparticle dissolution-based chemiluminescence (CL) method for immunoassay..$^{14}$

In this study, a novel method for high-sensitive biomolecule detection is demonstrated by using gold nanoparticles as signal amplification probes on a QCM. As the crucial points in the nanoparticle-based detection system, the conditions to maintain a good dispersion of the modified nanoparticles are investigated because nonspecific aggregation results in reduced sensitivity and selectivity. The modification of gold nanoparticles with biotin is performed by controlling the biotin density on the surfaces and the aggregation behaviors of the biotinylated gold nanoparticles are analyzed by UV-vis spectra and AFM images. Furthermore, we apply the sensing of streptavidin target molecules by a QCM device and use the biotinylated gold nanoparticles as signal amplification probes to improve the detection limit. The amplified frequency changes of the Auquartz crystal by the biotinylated gold nanoparticles are described and the dissipation changes are compared to provide new and unique information about the structure and properties of surface bound molecules.

\section{Experimental}

\section{Materials}

Gold nanoparticle dispersion (monodisperse, $20 \mathrm{~nm}$ average particle diameter), streptavidin from Streptomyces avidinii, bovine serum albumin (BSA), Tween 20, dimethyl sulfoxide (DMSO), (3-aminopropyl)triethoxysilane (APTES), and absolute ethanol were obtained from Sigma. Biotinylated bovine serum albumin, $N$-(6-[biotinamido]hexyl)-3'-(2'pyridyldithio)propionamide (biotin-HPDP), and dialysis membrane cassette (10000 MWCO) were purchased from Pierce (Rockford, IL). The etched silicon probes and $5 \mathrm{MHz}$ QCM sensors coated with gold were obtained from Veeco Metrology Group (Santa Barbara, CA) and Q-Sense (Gothenburg, Sweden), respectively.

\section{Preparation of the biotinylated gold nanoparticles}

We added $100 \mu \mathrm{l}$ of gold nanoparticle solution with a concentration of $0.8 \mathrm{nM}$ to $100 \mu \mathrm{l}$ of $1: 20$ dilution of the original PBS buffer ( $\mathrm{pH} 7.4$ ) containing $0.25 \%$ Tween 20 , and the mixture was allowed to stand for $30 \mathrm{~min}$ for physisorption of Tween 20 onto the surface of the gold nanoparticles. The surfactant Tween 20 was used in our system to prevent irreversible particle aggregation and to improve the long-term stability of gold nanoparticles. ${ }^{15}$ Then, various volumes of 4 $\mathrm{mM}$ biotin-HPDP dissolved in DMSO were added and the biotinylation of gold nanoparticles was carried out for $4 \mathrm{~h}$ at room temperature. Excess biotin-HPDP was removed from the surface-modified gold nanoparticles by dialysis against the diluted PBS buffer containing Tween 20 for $12 \mathrm{~h}$. The optical absorbance spectra of solutions obtained were investigated with a UV-vis spectrophotometer, using $1 \mathrm{~cm}$ path length quartz cuvettes. Atomic force microscopy (AFM) images were taken in tapping mode on a Multimode Nanoscope IV (Digital Instrument, Veeco Metrology Group, Santa Barbara, CA) using etched silicon probes. Images were recorded in height mode, and Nanoscope IV software was used for data processing.

\section{Quartz crystal microbalance (QCM) technique}

A quartz crystal microbalance with dissipation monitoring (QSense AB, Sweden) was used to verify signal amplification by

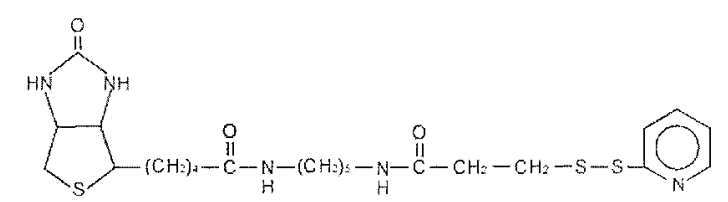

Scheme 1 The chemical structure of biotin-HPDP.

gold nanoparticles. The QCM apparatus allows for subsequent measurements of up to 4 harmonics of $5,15,25$, and $35 \mathrm{MHz}$, corresponding to the overtones $n=1,3,5$, and 7, respectively. ${ }^{16}$ In the present work, we used polished AT-cut crystals $(25 \mathrm{~mm}$ diameter) with a fundamental resonant frequency of $5 \mathrm{MHz}$; here the frequency shift and the dissipation shift for the third overtone are presented. The Sauerbrey sensitivity of $5 \mathrm{MHz}$ QCM crystals is $1 \mathrm{~Hz}=17.7 \mathrm{ng} / \mathrm{cm}^{2}$. The chips were cleaned first with a piranha solution consisting of a $3: 1$ ratio of $50 \%$ (w/v) aqueous solutions of $\mathrm{H}_{2} \mathrm{SO}_{4}$ and $30 \%$ (w/v) $\mathrm{H}_{2} \mathrm{O}_{2}$ (caution: this mixture reacts violently with organic materials and must be handled with extreme care), followed by a UV-ozone treatment. The cleaned chips were rinsed with absolute ethanol and dried with nitrogen. The fluid cell was temperature-stabilized to $22 \pm$ $0.1^{\circ} \mathrm{C}$. For sensing of streptavidin target molecules by QCM, the surfaces were calibrated with the diluted PBS buffer $(\mathrm{pH}$ 7.4) and activated by exposure to a $1: 1$ mixture of $0.1 \mathrm{mg} / \mathrm{ml}$ biotin-BSA and $0.1 \mathrm{mg} / \mathrm{ml} \mathrm{BSA}$ in the same buffer solution. At saturation, the surfaces were rinsed, and the streptavidin target molecules with various concentrations in the diluted PBS buffer were applied. In the signal amplification step, the dialyzed biotinylated gold nanoparticle solution was added to streptavidin target molecules bound to the sensing surfaces, and the changes of frequency and dissipation were monitored.

\section{Results and Discussion}

The colloidal gold nanoparticles were functionalized with biotin-HPDP that has an extended spacer arm (Scheme 1). The spacer arm can improve the complex formation of biotin with the deep biotin binding site of streptavidin and can increase the sensitivity of detection by streptavidin due to reduction of steric hindrance. Ligand-conjugated gold nanoparticles need to maintain good dispersion and long-term stability for use as signal amplification probes because their nonspecific aggregation results in reduced sensitivity and selectivity. Several methods have been used to improve the dispersivity in aqueous solution by utilizing surfactants and polymer additives. ${ }^{15,17}$ An alternative approach to obtain stabilized gold nanoparticles is to control the ligand density on the surface of the gold nanoparticles. ${ }^{18}$ With various biotin concentrations, we investigated the aggregation behavior of the gold nanoparticles by observing the solution colors and by monitoring the UV-vis spectra. Figure 1 shows representative UV-vis spectra of gold nanoparticles modified with biotin of different concentrations. The bare gold nanoparticle solutions were initially a pinkish-red color due to the well-dispersed nature of the particles and the surface plasmon resonance peak of the gold nanoparticles occurred at $520 \mathrm{~nm}$. Upon chemisorption of biotin-HPDP, the surface plasmon band underwent a redshift to $523-525 \mathrm{~nm}$ by the formation of dielectric layers around the colloidal gold. ${ }^{19}$ The exposure of gold nanoparticles to $20 \mu \mathrm{M}$ biotin-HPDP in the diluted PBS buffer containing $0.25 \%$ Tween 20 resulted in broadening of the spectrum and in the formation of a new 


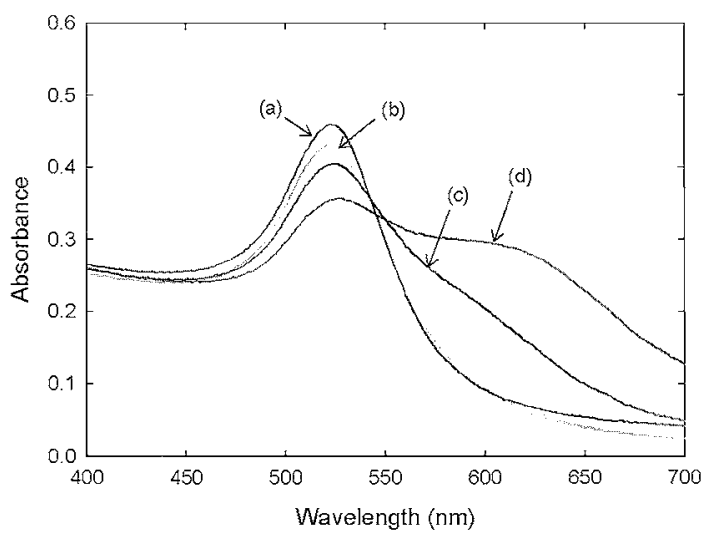

Fig. 1 Optical absorption spectra of the gold nanoparticles modified with biotin-HPDP of different concentrations: (a) bare gold nanoparticles, (b) $10 \mu \mathrm{M}$ biotin-HPDP + gold nanoparticles, (c) 15 $\mu \mathrm{M}$ biotin-HPDP + gold nanoparticles, and (d) $20 \mu \mathrm{M}$ biotin-HPDP + gold nanoparticles.

absorption band at wavelengths longer than $600 \mathrm{~nm}$, indicating particle aggregation. ${ }^{20}$ The modification of the gold nanoparticles with biotin-HPDP results in a net loss of the negative charge, which is the most important factor stabilizing colloidal gold preparations, and thus nanoparticle aggregation occurs because of decreased electrostatic repulsion. ${ }^{21}$ In the case of functionalization with $15 \mu \mathrm{M}$ biotin-HPDP, the biotinylated gold nanoparticles maintained a good suspension for several hours, but the color of the solution having gold nanoparticles changed from red to purple during storage at $4^{\circ} \mathrm{C}$. In contrast, the spectrum of a solution of gold nanoparticles biotinylated with $10 \mu \mathrm{M}$ biotin-HPDP was almost the same as that of a bare gold nanoparticle solution and did not show broadening of the spectrum or formation of a new absorption band. This clearly indicates that the biotinylated gold nanoparticles have good dispersivity in solution without aggregation. In addition, the gold nanoparticles modified with $10 \mu \mathrm{M}$ biotin-HPDP showed good long-term stability at $4^{\circ} \mathrm{C}$. From these results, we believe that gold particle aggregation depends on the coverage of the monolayer formed on the colloidal gold surface and that $10 \mu \mathrm{M}$ biotin-HPDP is the critical concentration to prevent aggregation of gold nanoparticles in our system. To confirm the biotinylation of gold particles, we investigated the streptavidin-induced aggregation of gold nanoparticles by AFM image analysis. To immobilize the biotinylated gold nanoparticles and the streptavidin-induced gold nanoparticle aggregates, we used APmica, which was obtained by exposure of freshly cleaved mica to a very low concentration of APTES in the vapor. ${ }^{22}$ Figure $2 \mathrm{~A}$ shows a typical AFM image of the gold nanoparticles biotinylated with $10 \mu \mathrm{M}$ biotin-HPDP; the particles are well separated from each other. However, the biotinylated gold nanoparticles were observed to be mostly aggregated after the addition of streptavidin target molecules (Fig. 2B). The streptavidin-induced gold nanoparticle aggregates were prepared by adding $10 \mu \mathrm{l}$ of $0.1 \mathrm{mg} / \mathrm{ml}$ streptavidin to $200 \mu \mathrm{l}$ of the dialyzed biotinylated gold nanoparticles and then incubating the mixture for $30 \mathrm{~min}$ at room temperature.

In a subsequent set of experiments, the gold nanoparticles functionalized with $10 \mu \mathrm{M}$ biotin-HPDP were chosen as signal amplification probes for sensing the streptavidin target molecules by QCM. The QCM technique ${ }^{23}$ is a high-resolution mass sensing technique in which an increase in mass bound to
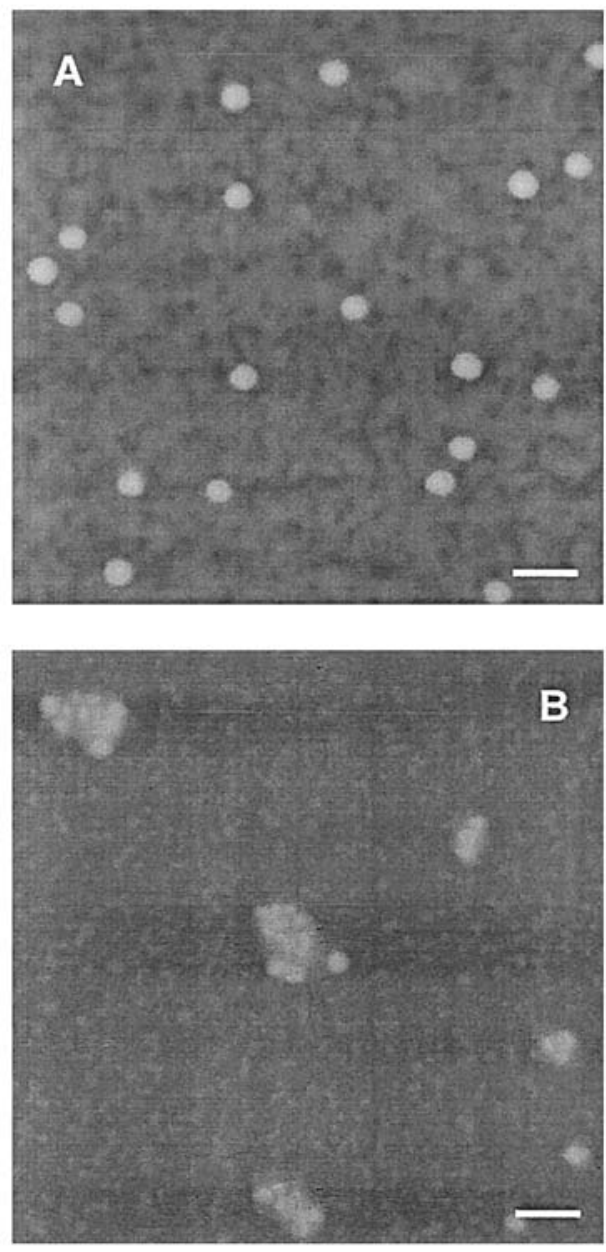

Fig. 2 AFM images of (A) the gold nanoparticles modified with 10 $\mu \mathrm{M}$ biotin-HPDP, and (B) the gold nanoparticles that have been aggregated by adding streptavidin to the biotinylated gold nanoparticle solution. Scale bars correspond to $100 \mathrm{~nm}$.

the quartz surface causes the crystal's resonant frequency to decrease, according to the Sauerbrey relation:

$$
\Delta m_{\mathrm{QCM}}=\left(C_{\mathrm{QCM}} / n\right) \Delta f
$$

where $C_{\mathrm{QCM}}\left(=17.7 \mathrm{ng} \mathrm{cm}{ }^{-2} \mathrm{~Hz}^{-1}\right.$ at $\left.f=5 \mathrm{MHz}\right)$ is the mass sensitivity constant and $n(=1,3, \ldots)$ is the overtone number. In this way, effective strategies for mass enhancement have been developed for high-sensitive detection of target molecules with very low concentrations. ${ }^{24}$ The general concept for the amplified detection of streptavidin target molecules in this study is as follows: the biotinylated BSA is first adsorbed on the $\mathrm{Au}$ surface of the QCM electrode; the subsequent coupling of the streptavidin target molecules to the biotin-BSA in the sensing interface is then carried out; and amplification of the sensing process is performed by the interaction of the biotinylated gold nanoparticles with the streptavidin target molecules on the sensing surface. Figure 3 shows the time-dependent frequency changes of the Au-quartz crystal for exposure of the biotinylated BSA layer to streptavidin, followed by the biotinylated gold nanoparticles. The adsorption of the mixture, consisting of $0.1 \mathrm{mg} / \mathrm{ml}$ of biotinylated BSA and $0.1 \mathrm{mg} / \mathrm{ml}$ of BSA, on the gold surface resulted in a frequency shift of $c a .4 .6$ $\mathrm{Hz}$ and the leveling-off of the frequency to a constant value after $20 \mathrm{~min}$. The BSA mixture with higher concentrations did 


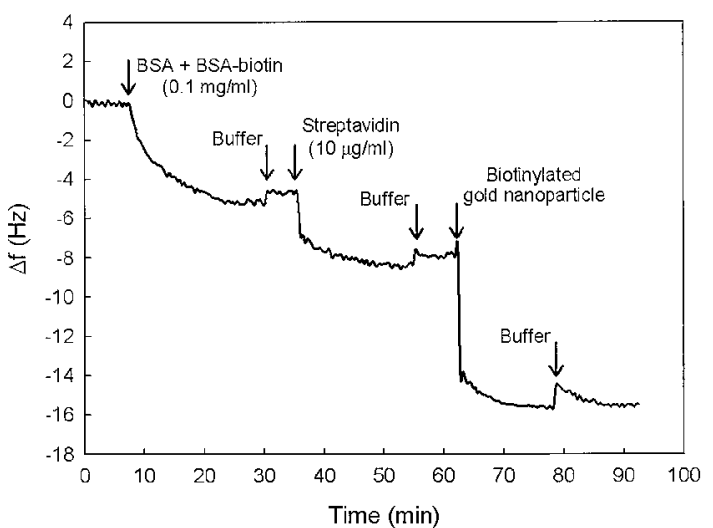

Fig. 3 Changes in frequency versus time upon the sequential exposure of the Au-quartz crystal to the biotinylated BSA, streptavidin, and the biotinylated gold nanoparticles.

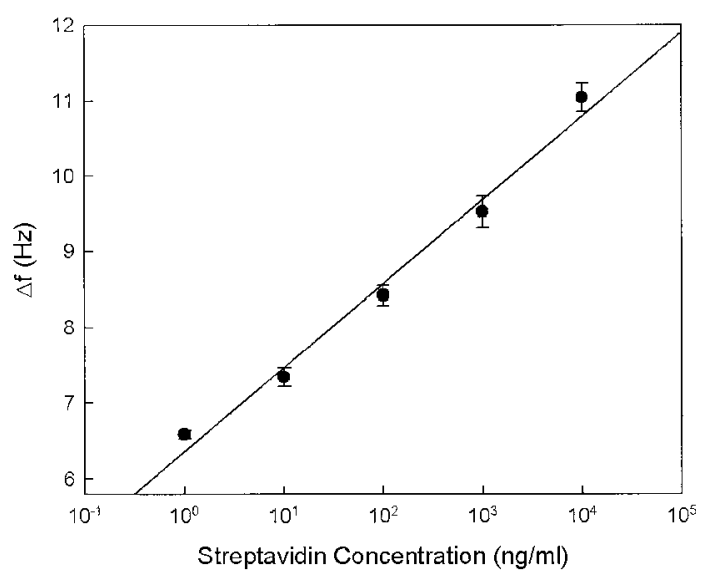

Fig. 4 The calibration curve determined from the net frequency changes upon signal amplification by biotinylated gold nanoparticles. The concentration of streptavidin target molecules ranged from 1 $\mathrm{ng} / \mathrm{ml}$ to $10 \mu \mathrm{g} / \mathrm{ml}$. The error bars represent standard deviations (SD) of $n=3$.

not increase the crystal frequency change, implying that the gold surface was saturated. The target streptavidin was subsequently bound to the biotinylated BSA and a frequency change of only $3.3 \mathrm{~Hz}$ was observed after $20 \mathrm{~min}$ exposure. However, treatment of the resulting interface with the biotinylated gold nanoparticles enhanced the sensing signal with an additional frequency change of $c a .7 .8 \mathrm{~Hz}$, indicating that the binding of the gold nanoparticles amplified the sensing of the streptavidin target molecules due to the mass increase. Figure 4 shows the calibration curve determined from the net frequency changes of frequency changes by streptavidin target molecules with different concentrations and additional frequency changes due to biotinylated gold nanoparticles. The biotin-BSA-coated $\mathrm{Au}$ surfaces were treated with streptavidin target molecules in the range of $1 \mathrm{ng} / \mathrm{ml}-10 \mu \mathrm{g} / \mathrm{ml}$ and the resulting frequency changes were in the range from 0.39 to $3.3 \mathrm{~Hz}$. The detection limit was assessed to be $50 \mathrm{ng} / \mathrm{ml}$, which was calculated according to the IUPAC signal to noise guideline $(S / N=3){ }^{25}$ However, the signal amplification process by biotinylated gold nanoparticles yielded additional frequency changes corresponding to $6.2-7.8 \mathrm{~Hz}$ in the range from $1 \mathrm{ng} / \mathrm{ml}$ to 10 $\mu \mathrm{g} / \mathrm{ml}$. The calibration curve determined from the net frequency changes showed good linearity over a wide range from $1 \mathrm{ng} / \mathrm{ml}$

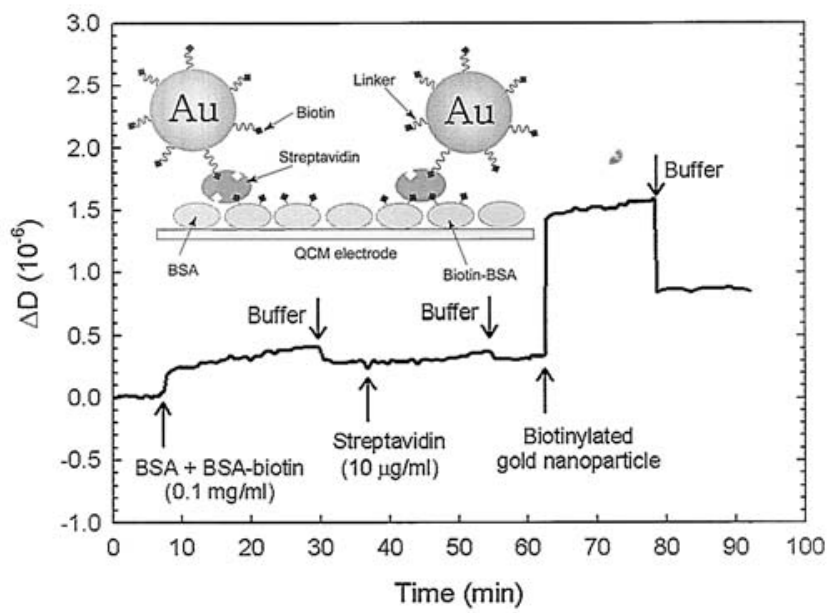

Fig. 5 Changes in energy dissipation versus time obtained from the QCM measurements for the same sequence of events as displayed in Fig. 3. Inset shows the schematic illustration of the structure of the layers formed on the Au electrode by the binding reactions including adsorption of the biotin-BSA solution to the Au electrode, binding of streptavidin target molecules to the biotin-BSA layer, and the subsequent association of the biotinylated gold nanoparticles.

to $10 \mu \mathrm{g} / \mathrm{ml}$, the working range for the quantitative streptavidin target molecule analysis. These experiments clearly indicate that gold nanoparticles can be used as signal amplification probes to improve the detection limits of conventional detection systems.

In addition, the simultaneously recorded dissipation changes reveal the structure or properties of surface-bound molecules. The dissipation change is measured by periodically switching off the driving power to the thickness-shear-mode oscillation of the sensor crystal and recording the decay of the damped oscillation. The decrease in shear stiffness of the layer or the lack of compact structure results in a $\Delta D$ increase because it dampens the oscillation. ${ }^{26}$ Figure 5 shows the dissipation changes versus time from the QCM measurements for the same sequence of events as displayed in Fig. 3. Adsorption of the biotin-BSA solution to the $\mathrm{Au}$ electrode resulted in slight dissipation increases of $0.3 \times 10^{-6}$; no additional energy dissipation was induced during binding of streptavidin target molecules to the biotin-BSA layer. However, the subsequent association of the biotinylated gold nanoparticles was accompanied by a relatively large dissipation shift of $1.2 \times 10^{-6}$. The observed significant dissipation changes suggest that the layer of biotin-BSA adsorbed on the Au electrode forms a compact structure and that the streptavidin layer assembled on the biotin-BSA surface is highly compact and rigid, while the structure formed by the biotinylated gold nanoparticles on the streptavidin layer is flexible and dissipative, being elongated outward from the sensing surface.

\section{Conclusion}

We have demonstrated a novel strategy for signal amplification on a QCM device by using the gold nanoparticle-promoted mass enhancement. By employing a streptavidin-biotin interaction as a model system, we performed the modification of gold nanoparticles with biotin by controlling the biotin density on the surfaces, because nonspecific aggregation generally results in reduced sensitivity and selectivity. The aggregation 
behavior of biotinylated gold nanoparticles was investigated by UV-vis spectra and AFM images. For sensing streptavidin target molecules by QCM, gold nanoparticles could be used as signal amplification probes to improve the detection limit of conventional detection systems. The calibration curve determined for the net frequency changes showed good linearity over a wide range from $1 \mathrm{ng} / \mathrm{ml}$ to $10 \mu \mathrm{g} / \mathrm{ml}$ for the quantitative analysis of streptavidin target molecules. The simultaneously recorded dissipation changes reveal that the structure formed by the biotinylated gold nanoparticles on the streptavidin layer is flexible and dissipative, being elongated outward from the sensing surface. We expect that this approach may be extendable to the high-sensitive detection of various biomolecules. In particular, the measurement of dissipation changes can provide new and unique information about the structure and properties of the layers formed on the $\mathrm{Au}$ electrode of a QCM. In future work based on this study, other nanoparticles will be studied as signal enhancement probes for high-sensitive detection.

\section{Acknowledgements}

This work was supported by the Korea Research Foundation Grant funded by the Korean Government (KRF-2004-205D00065) and the research fund of Hanyang University (HY2003).

\section{References}

1. C. M. Niemeyer and C. A. Mirkin (ed.), "Nanobiotechnology: Concepts, Applications, and Perspectives", 2004, Wiley-VCH, Weinheim.

2. C. M. Niemeyer, Angew. Chem., Int. Ed., 2001, 40, 4128.

3. I. Willner and E. Katz, Angew. Chem., Int. Ed., 2003, 42, 4576.

4. T. A. Taton, G. Lu, and C. A. Mirkin, J. Am. Chem. Soc., 2001, 123, 5164.

5. N. T. K. Thanh and Z. Rosenzweig, Anal. Chem., 2002, 74,
1624.

6. Y. Xiao, F. Patolsky, E. Katz, J. F. Hainfeld, and I. Willner, Science, 2003, 299, 1877.

7. A. Perrin, V. Lanet, and A. Theretz, Langmuir, 1997, 13, 2557.

8. N. Levit-Binnun, A. B. Lindner, O. Zik, Z. Eshhar, and E. Moses, Anal. Chem., 2003, 74, 1436.

9. S. Li, P. He, J. Dong, Z. Guo, and L. Dai, J. Am. Chem. Soc., 2005, 127, 14.

10. T. A. Taton, C. A. Mirkin, and R. L. Letsinger, Science, 2000, 289, 1757.

11. J. Wang, D. Xu, A.-N. Kawde, and R. Polsky, Anal. Chem., 2001, 73, 5576.

12. L. He, M. D. Musick, S. R. Nicewarner, F. G. Salinas, S. J. Benkovic, M. J. Natan, and C. D. Keating, J. Am. Chem. Soc., 2000, 122, 9071.

13. D. S. Grubisha, R. J. Lipert, H.-Y. Park, J. Driskell, and M. D. Porter, Anal. Chem., 2003, 75, 5936.

14. A. Fan, C. Lau, and J. Lu, Anal. Chem., 2005, 77, 3238.

15. K. Aslan and V. H. Perez-Luna, Langmuir, 2002, 18, 6059.

16. F. Hook, B. Kasemo, T. Nylander, C. Fant, K. Sott, and H. Elwing, Anal. Chem., 2001, 73, 5796.

17. Y. Kim, R. C. Johnson, and J. T. Hupp, Nano Lett., 2001, 1, 165.

18. R. Jin, G. Wu, Z. Li, C. A. Mirkin, and G. C. Schatz, J. Am. Chem. Soc., 2003, 125, 1643.

19. N. Nath and A. Chilkoti, Anal. Chem., 2002, 74, 504.

20. K. Aslan, J. R. Lakowicz, and C. D. Geddes, Anal. Biochem., 2004, 330, 145.

21. C. S. Weisbecker, M. V. Merritt, and G. M. Whitesides, Langmuir, 1996, 12, 3763.

22. G. H. Seong, T. Niimi, Y. Yanagida, E. Kobatake, and M. Aizawa, Anal. Chem., 2000, 72, 1288.

23. K. A. Marx, Biomacromolecules, 2003, 4, 1099.

24. I. Willner, F. Patolsky, Y. Weizmann, and B. Willner, Talanta, 2002, 56, 847.

25. X. C. Zhou, S. J. O'Shea, and S. F. Y. Li, Chem. Commun., 2000, 953.

26. X. Su, Y. Wu, R. Robelek, and W. Knoll, Langmuir, 2005, 21,348 . 